\title{
Hemostatic Abnormalities and Liver Diseases
}

\author{
Hideo Wada, M.D., Ph.D., ${ }^{1}$ Masanobu Usui, M.D., Ph.D., ${ }^{2}$ \\ and Nobuo Sakuragawa, M.D., Ph.D. ${ }^{3,4}$
}

\section{ABSTRACT}

Professor Eberhard F. Mammen greatly contributed to the understanding of the relationship between hemostatic abnormalities and liver diseases. The physiology of the hemostatic system is closely linked to liver function because the liver parenchymal cells produce most of the factors of the clotting and fibrinolytic systems. Acute or chronic hepatocellular diseases and hepatic failure including liver cirrhosis, vitamin $\mathrm{K}$ deficiency, liver surgery including liver transplantation, and sclerotherapy of bleeding esophageal varices, which were classified by Prof. Mammen, show various hemostatic abnormalities in the coagulation system, fibrinolytic system, platelets, and the reticuloendothelial system. Hemostatic abnormalities in patients with hepatic failure or in those that have undergone liver surgery are similar to those in disseminated intravascular coagulation. Prof. Mammen also contributed to the study of vitamin $\mathrm{K}$-dependent clotting factors, antithrombin, and hemostatic molecular markers. Partly based on this work, the prothrombin time-international normalized ratio, several hemostatic molecular markers, and antithrombin therapy have been recently developed for the diagnosis and treatment of thrombosis.

KEYWORDS: Liver disease, hemostasis, vitamin K deficiency, disseminated intravascular coagulation

The physiology of the hemostatic system is closely linked to liver function because the liver parenchymal cells produce most of the factors of clotting and of the fibrinolytic systems and because the liver regulates the activation or inhibition of both systems. ${ }^{1}$ Professor Eberhard F. Mammen greatly contributed to the understanding of the relationship between hemostatic abnormalities and liver diseases. ${ }^{1-4} \mathrm{~A}$ disturbed liver parenchymal cell function adversely impacts the hemostasis system, the extent of which correlates with the degree of disease. These changes may be modest in patients with mild liver disease but are severe in patients with grossly compromised liver function. A wide spectrum of hemostatic defects is found in patients with liver cirrhosis. Thrombocytopenia and thrombocytopathy usually complicate the clinical presentation in patients with liver cirrhosis, and systemic activation of the fibrinolytic system is always seen in cirrhotic individuals. The reticuloendothelial system (RES) of the liver greatly participates in the clearance of breakdown products of activated clotting factors such as fibrin-related products, thrombin-antithrombin complex (TAT), plasmin-plasmin-inhibitor complex (PPIC), and activated platelets. Prof. Mammen was an early pioneer in the investigation of various hemostatic abnormalities associated with many liver diseases including liver cirrhosis, and several liver abnormalities due to liver transplantation ${ }^{5}$ or bone marrow transplantation ${ }^{6}$ have been recently noted.
${ }^{1}$ Departments of Molecular and Laboratory Medicine, and ${ }^{2}$ First Department of Surgery, Mie University Graduate School of Medicine, Tsu-city, Japan; ${ }^{3}$ Toyama University, School of Medicine, Toyama City, Toyama, Japan; ${ }^{4}$ Toyoura Hospital, Toyoura, Japan.

Address for correspondence and reprint requests: Prof. Hideo Wada, M.D., Ph.D., Department of Laboratory Medicine, Mie University School of Medicine, 2-174 Edobashi, Tsu-city, Mie-ken
514-8507, Japan (e-mail: wadahide@clin.medic.mie-u.ac.jp)

A Tribute to Eberhard F. Mammen, M.D. (1930-2008); Guest Editor, Emmanuel J. Favaloro, Ph.D., M.A.I.M.S.

Semin Thromb Hemost 2008;34:772-778. Copyright (C) 2008 by Thieme Medical Publishers, Inc., 333 Seventh Avenue, New York, NY 10001, USA. Tel: +1(212) 584-4662.

DOI 10.1055/s-0029-1145259. ISSN 0094-6176. 


\section{HEMOSTATIC FACTORS PRODUCED BY THE LIVER}

With few exceptions, such as von Willebrand factor (VWF), tissue-type plasminogen activator (tPA), plasminogen activator inhibitor-1 (PAI-1), thrombomodulin (TM), and urokinase-type plasminogen activator (uPA), all other coagulation proteins and inhibitors are either solely or partially synthesized by liver parenchymal cells $^{1-3}$ (Table 1).

\section{Vitamin K-Dependent Factors}

Four procoagulant factors of the clotting system (factors II, VII, IX, and X), two anticoagulant factors (protein C and protein $S$ ), and protein $Z$ require post-ribosomal modification after synthesis for proper physiologic function. These so-called vitamin K-dependent factors possess a specific number of glutamic acid residues that function as potential binding sites for calcium bridges in the $\mathrm{NH}_{2}$-terminal region. The glutamic acid residues are converted to $\gamma$-carboxyglutamic acids, and this modification is facilitated by a carboxylase that requires the fat-soluble vitamin $\mathrm{K}$ as a cofactor. Both vitamin $\mathrm{K}$ epoxide reductase (VKOR) and $\gamma$-glutamyl carboxylase exist in the liver. ${ }^{7}$ A failure of carboxylation, as in any form of vitamin K deficiency or during therapy with oral anticoagulants, will yield proteins (proteins induced by vitamin $\mathrm{K}$ absence; PIVIKA) that cannot be bound by calcium bridges to structural phospholipid surfaces, a prerequisite for activation complex formation such as tenase complexes, prothrombinase complexes, and extrinsic pathway complex. PIVIKA-II is also a tumor marker of hepatocellular carcinoma. ${ }^{8}$ The resulting impaired functioning of the clotting system is often reflected by changes in the activated partial thromboplastin time (APTT), prothrombin time (PT), thrombin time (TT), hepaplastin test, and thrombo test. Hemostatic defects, especially of factor VII (FVII), thus depend on the extent of residual liver function. Therefore, liver function is now evaluated using the $\mathrm{PT}^{9}$ and hepaplastin test.

\section{Other Factors}

Other clotting factor such as fibrinogen, FV, FVIII, FXI, FXII, prekallikrein, and high-molecular-weight kininogen, proteins in the fibrinolytic system such as plasminogen and $\alpha 2$-antiplasmin inhibitor, plasmin inhibitor (PI) and anticoagulant protease inhibitors such as antithrombin (AT) ${ }^{10}$ heparin cofactor $\mathrm{II},{ }^{11}$ protein C (PC), protein $\mathrm{S}(\mathrm{PS})$, and tissue factor pathway inhibitor (TFPI) are also synthesized by the liver.

Prof. Mammen reviewed AT in detail. ${ }^{12,13}$ AT is a single-chain glycoprotein in plasma and belongs to the family of the serpins. It is synthesized in liver parenchymal cells, and its plasma concentration is between 112 and $140 \mathrm{mg} / \mathrm{L}$. AT is a unique inhibitor of the clotting system and neutralizes most of the enzymes generated during activation of the clotting cascade, especially thrombin, FXa, and FIXa. Equimolar, irreversible complexes are formed between $\mathrm{AT}$ and the enzymes including TAT. A TAT assay has been developed as a sensitive marker for thrombosis such as predisseminated intravascular coagulation (DIC). ${ }^{14}$ The interaction between AT and the activated clotting factors is at least 1000-fold increased in the presence of heparin and its derivatives. Heparins bind to multiple sites of the AT molecule, resulting in a steric reconfiguration. Heparins contain a specific pentasaccharide unit that is the minimum requirement for AT binding. The glycosaminoglycan (GAG) heparan sulfate found on endothelial cell surfaces also contains this pentasaccharide and can thus "activate" AT. It is traditionally acknowledged that much of the physiologic inactivation of enzymes by AT occurs on the endothelium, mediated by heparan sulfate. The binding of AT to the GAGs also releases prostacyclin, which possesses strong antiinflammatory properties. ${ }^{15}$ In a clinical trial of highdose AT treatment for severe sepsis, ${ }^{16}$ AT significantly reduced the mortality in patients treated without heparin or in those with DIC. ${ }^{17}$ Decreased AT concentration in plasma is rarely due to genetic abnormalities ${ }^{18}$ but is usually acquired in patients with liver disorders or those with DIC. ${ }^{12}$ In patients with DIC, especially due to sepsis, increased consumption of AT is frequently observed. During acute and severe DIC, clotting factors and inhibitors are consumed faster than they can be reproduced. Therefore, the observation of reduced plasma AT levels not only helps the diagnosis but also predicts outcome in patients with septic DIC. ${ }^{19}$ The important role of AT in DIC and sepsis is the basis for considering AT concentrates as an additional therapeutic modality. ${ }^{12,16,17}$

Table 1 Hemostatic Factors Synthesized by the Liver

\begin{tabular}{|c|c|c|}
\hline & Factor & Inhibitor \\
\hline Coagulation system & $\begin{array}{l}\text { Fibrinogen, FII, FV, FVII, FVIII, FIX, FX, FXI, } \\
\text { FXII, FXIII, prekallikrein, high-molecular-weight } \\
\text { kininogen }\end{array}$ & AT, PC, PS, TFPI, heparin cofactor II \\
\hline Fibrinolytic system & Plasminogen & $\mathrm{PI}$ \\
\hline Platelets & (Thrombopoietin) & ADAMTS13 \\
\hline
\end{tabular}


In the fibrinolytic system, Prof. Mammen and his co-workers reported two families and one single patient with a heterozygous PI deficiency that is a rare congenital bleeding disorder. ${ }^{20}$ All patients and most relatives with the defect had a mild bleeding tendency. Mammen and colleagues suggested the incorporation of the PI assay for all patients who have a bleeding disorder with normal platelet studies and normal clotting profiles. After the study of PI, the PPIC assay ${ }^{21}$ was developed to measure plasmin generation. The assay of PI or PPIC is useful to monitor anti-fibrinolytic therapy or fibrinolytic therapy such as that using tPA or uPA.

Hemostatic factors such as thrombopoietin ${ }^{22}$ and a disintegrin-like and metalloproteinase with thrombospondin type 1 motifs 13 (ADAMTS13) ${ }^{23}$ in platelets were recently reported to be synthesized by the liver. Thrombopoietin promotes thrombopoiesis, regulates the number of platelets in bone marrow, and is reduced after liver resection, and this thus causes thrombocytopenia. ${ }^{24}$ ADAMTS13 cleaves the ultralarge multimers of VWF to prevent hyperaggregation of platelets, and the deficiency of ADAMTS13 $3^{25}$ is known to cause thrombotic thrombocytopenic purpura (TTP). Thrombotic microangiopathy (TMA) is sometimes observed during liver transplantation, and reduction of ADAMTS13 is thought to be one of the important causes of TMA. ${ }^{26}$

\section{HEMOSTATIC ABNORMALITIES DUE TO VARIOUS LIVER DISEASES}

Prof. Mammen identified many hemostatic abnormalities associated with various liver diseases after several types of liver disturbance ${ }^{1-3}$ (Table 2). Acute or chronic hepatocellular diseases, liver cirrhosis, vitamin K deficiency, liver surgery including liver transplantation, and sclerotherapy of bleeding esophageal varices are associated with various hemostatic abnormalities, which involve the coagulation system, fibrinolytic system, platelets, and RES. Patients with hepatic failure including liver cirrhosis may present with the entire or wide spectrum of factor deficiencies and may even develop a peculiar form of DIC. ${ }^{27,28}$ Except for FVIII:C and VWF, all procoagulant and inhibitory factors are reduced, as a result of impaired protein synthesis. Abnormal fibrinogen ${ }^{29}$ and prothrombin molecules can be identified, and both the platelet count and function are altered.

Prof. Mammen and co-workers developed a fully automated procedure for the determination of fibrin( $\mathrm{o}^{-}$ gen) degradation products (FDPs) known as the I. L. Multistat Centrifugal Analyzer (MCA; Instrumentation Laboratories, Lexington, MA). ${ }^{30}$ The assay is based on the turbidometric measurement of the interaction between antibodies and human fibrinogen and defibrinogenated specimens. This method makes the accurate quantization of degradation products between 10 and $160 \mu \mathrm{g} / \mathrm{mL}$ possible with high reproducibility. The method can be adapted to existing automated coagulation procedures and can become part of coagulation panels. Recently, many fibrin-related markers such as soluble fibrin (SF) and D-dimer and fibrin and FDPs have been automatically measured, representing a valuable parameter for the diagnosis of deep vein thrombosis (DVT)/ pulmonary embolism $(\mathrm{PE})$ or DIC. ${ }^{31,32} \mathrm{D}$-dimer tends to be used to reduce the need for invasive and costly

Table 2 Laboratory Findings in Patients with Mild, Moderate, and Severe Hepatocellular Damage

\begin{tabular}{|c|c|c|c|c|}
\hline & \multirow[b]{2}{*}{ Test } & \multicolumn{3}{|c|}{ Hepatocellular Damage (Except with Surgery) } \\
\hline & & Mild & Moderate & Severe (Cirrhosis) \\
\hline \multirow[t]{8}{*}{ Coagulation system } & PT/APTT & $\mathrm{N}$ to $\uparrow$ & $\uparrow$ & $\uparrow$ \\
\hline & $\mathrm{TT}$ & N & $N$ to $\uparrow$ & $\uparrow$ \\
\hline & FVII & $\mathrm{N}$ to $\uparrow$ & $\downarrow$ & $\downarrow$ \\
\hline & FII, FIX, FX & $\mathrm{N}$ & $\downarrow$ & $\downarrow$ \\
\hline & VWF/FVIII & $\mathrm{N}$ to $\uparrow$ & $\uparrow$ & $\uparrow$ \\
\hline & Fibrinogen & $\mathrm{N}$ & $\downarrow$ & $\downarrow$ \\
\hline & FV, FXI, FXII & N & $\downarrow$ & $\downarrow$ \\
\hline & TAT & & & $\uparrow$ \\
\hline \multirow[t]{6}{*}{ Fibrinolytic system } & ELT & $\mathrm{N}$ & $\downarrow$ & $\downarrow$ \\
\hline & Fibrin(ogen) split products (FSPs) & $\mathrm{N}$ & $(\uparrow)$ & $\uparrow$ \\
\hline & Plasminogen/PI & $\mathrm{N}$ & $(\downarrow)$ & $\downarrow$ \\
\hline & tPA/uPA & $\mathrm{N}$ & $\mathrm{N}$ & $\mathrm{N}$ \\
\hline & PAl-1 & $\mathrm{N}$ & $N$ to $\downarrow$ & $\downarrow$ \\
\hline & PPIC & $\mathrm{N}$ & $\mathrm{N}$ to $\uparrow$ & $\uparrow$ \\
\hline \multirow[t]{2}{*}{ Platelets } & Bleeding time & $\mathrm{N}$ & $N$ & $\uparrow$ \\
\hline & Platelet count & $\mathrm{N}$ & & \\
\hline Platelets & Platelet function & $\mathrm{N}$ & $\mathrm{N}$ & $\downarrow$ \\
\hline
\end{tabular}

N, normal; $(\uparrow)$ or $(\downarrow)$, sometimes increased or decreased.

Source: Modified from Mammen EF. Coagulopathies of liver disease. Clin Lab Med 1994;14:769-780. 
imaging by exclusion of thrombosis when a D-dimer value below the method-specific cutoff is observed in the outpatient setting. ${ }^{32}$

\section{Acute or Chronic Hepatocellular Disease}

Patients with acute or chronic liver infection or with toxic or drug-induced hepatitis may have an impaired hemostatic system that can predispose the patient to bleeding. ${ }^{1-3}$ The degree of disturbance is related to the extent of liver parenchymal cell damage. Some patients with mild hepatitis may display no abnormal findings. Others may have slightly prolonged PT that can be traced back to slightly below normal levels of FVII, which with PC represents the most sensitive of all the vitamin $\mathrm{K}$-dependent factors. The plasma levels of other vitamin $\mathrm{K}$-dependent factors and non-vitamin-Kdependent factors may also be decreased. These changes are readily noticeable by prolonged PT and APTT, but the TT and fibrinogen levels are usually within the normal range. Abnormal fibrinogen and prothrombin molecules can also be identified, though less frequently. The levels of FVIII and VWF, which are acute inflammatory proteins, are usually elevated. All procoagulant and inhibitory factors, except for FVIII:C and VWF, are decreased, which is a reflection of impaired protein synthesis. Mild thrombocytopenia can be encountered in patients with hepatitis, but this appears to be clinically insignificant in bleeding, and currently platelet count is one of the most useful indicators for success of interferon therapy for hepatitis. ${ }^{33}$

\section{Liver Cirrhosis}

Patients with cirrhosis have a broad spectrum of hemostatic abnormalities involving the clotting system, the fibrinolytic system, and platelets. ${ }^{1-3}$ Because of the impaired protein synthesis, most factors and inhibitors of the clotting and the fibrinolytic systems are markedly reduced. In addition, abnormal vitamin $\mathrm{K}$-dependent factors and fibrinogen molecules are frequently encountered. Most patients have hyperfibrinolysis and thrombocytopenia, and nearly all patients have some bleeding tendency. Blood transfusion ${ }^{34}$ is sometimes required in severe bleeding. These changes are reflected in a progressively prolonged PT and APTT. As the procoagulant factors decrease, the regulators of the clotting system (AT, PC, and PS) also will decline. Fibrinogen levels are usually decreased, especially in severe forms of cirrhosis, and abnormal fibrinogen molecules ${ }^{29}$ can be detected. Plasminogen and PI are also affected by liver parenchymal cell damage and are usually below the normal range. In contrast, tPA, uPA, and PAI-1 levels usually remain unchanged, and evidence of an activated fibrinolytic system in many patients can be shown by shortened euglobulin lysis times (ELT) and elevated D-dimer. The mechanism of activated fibrinolysis is due to elevated levels of activators, especially tPA, resulting from the impaired clearance of activators and decreased levels of inhibitors, including histidine-rich glycoprotein (HRGP). ${ }^{35}$ Hemostatic molecular markers such as TAT, PPIC, and D-dimer are frequently elevated in patients with cirrhosis. Thrombocytopenia, due to pooling of platelets in an enlarged spleen, is a common feature of liver cirrhosis. In addition, increased consumption, folic acid deficiency, and decreased thrombopoiesis may contribute to the low platelet counts. Therefore, Prof. Mammen suggested that severe liver cirrhosis has laboratory findings similar to those of DIC, indicating that a differential diagnosis between DIC and severe liver failure is still challenging. ${ }^{36}$

\section{Vitamin K Deficiency}

Hepatobiliary diseases such as obstruction and fistulas, intravenous nutrition, and prolonged use of certain oral antibiotics may lead to vitamin $\mathrm{K}$ deficiency. ${ }^{1-3}$ The hemostatic defects related to vitamin $\mathrm{K}$ deficiency are limited to the vitamin $\mathrm{K}$-dependent procoagulants and anticoagulants (FII, FVII, FIX, FX, and PC and PS); all other parameters remain in the normal range. The drop in the levels of PC parallels the decline in FVII. Therefore, measurement of both FVII and PC is useful for the diagnosis of $\mathrm{PC}$ deficiency in patients treated with oral anticoagulant therapy. ${ }^{37}$ The decrease in procoagulants will initially result in a slightly prolonged PT and ultimately in a markedly abnormal PT and APTT. All other screening tests and clotting factor levels are normal. These factors indicate that the monitoring of oral anticoagulant therapy by prothrombin time-international normalized ratio (PT-INR) is useful for anticoagulation therapy. ${ }^{38}$ An examination of VKOR and cytochrome P450 2C9 (CYP2C9) genes may be helpful in determining the initial amount of coumarins and the individual response for adjusting the dosage accordingly. ${ }^{39,40}$

\section{Liver Surgery}

Liver transplantation has become an established procedure for patients with severe, life-threatening liver disease, especially in patients with end-stage hepatitis B infection. In addition to orthotopic liver transplantation, patients are subjected to partial liver resections and peritoneovenous (LeVeen) shunts (Table 3). Surgery associated with the liver leads to major hemostatic alterations. ${ }^{1-3}$ The disturbances associated with liver surgery depend on the underlying liver problem, surgical procedure, and host reaction. Prof. Mammen and his $\mathrm{co}^{-}$ workers studied AT, prekallikrein, and fibronectin levels in 290 surgical patients. ${ }^{41}$ The AT $(49 \% \pm 17)$, prekallikrein $(26 \% \pm 12)$, and fibronectin $(128 \mu \mathrm{g} / \mathrm{mL} \pm 72)$ 


\begin{tabular}{|c|c|c|c|}
\hline & \multicolumn{3}{|c|}{ Operation } \\
\hline & $\begin{array}{l}\text { Peritoneovenous } \\
\text { Shunt (LeVeen) }\end{array}$ & Partial Liver Resection & $\begin{array}{l}\text { Orthotopic Liver } \\
\text { Transplantation }\end{array}$ \\
\hline \multirow[t]{6}{*}{ Cause for bleeding } & DIC & Underlying diseases & Underlying diseases \\
\hline & & Mechanical bleeding & Mechanical bleeding \\
\hline & & Consumption & Consumption \\
\hline & & $\mathrm{DIC}$ & Primarily fibrinolysis \\
\hline & & & DIC \\
\hline & & & TMA \\
\hline
\end{tabular}

levels in septic patients were significantly lower than those in the patients without sepsis. The AT level in patients who died $(42 \% \pm 22)$ was significantly lower than that in patients who survived. They suggested that AT, prekallikrein, and fibronectin levels in critically ill surgical patients might allow earlier diagnosis and more effective treatment of sepsis.

Bleeding associated with a partial liver resection is mostly mechanically induced, but chronic DIC may also be present. Orthotopic liver transplantation can be associated with severe hemorrhage, which is partly due to the preexisting hemostasis defects and partly to a form of DIC with a marked fibrinolytic response. This is especially noted during the anhepatic phase and when the donor liver is perfused by the recipient's blood. Recently hemostatic abnormalities during liver transplantation have been classified into five periods ${ }^{42}$ : preoperative period, preanhepatic stage, anhepatic phase, reperfusion and post-reperfusion phase, and postoperative period. Postoperative recovery is quick, provided the graft is not rejected. Postoperatively, there may be an initial hypercoagulable state, which could be related to the thrombosis occasionally encountered. ${ }^{43,44}$ TMA is also reported to be one of the most important complications in liver transplantation. ${ }^{5,45}$

Perioperative defects in the hemostasis system can be extensive and depend on the underlying disease. They usually resemble those illustrated in Table 4 . The first major hemorrhages are encountered when the patient's liver has been removed and when the patient is in an anhepatic phase. There is a significant activation of the fibrinolytic system, apparently due to a major release of tPA. This fibrinolytic "burst" is apparently due to a massive release of tPA from the patient's endothelial cells and a reduction in phagocytosis capacity of the RES. A second fibrinolytic burst arises as the donor liver is perfused. Once again, extremely high tPA levels and very low PAI-1 levels can be found. Some of the laboratory findings in DIC may be a reflection of decreased hepatic clearance of activation products by the RES of the diseased liver. Severe bleeding due to the activation of the fibrinolytic system is encountered in orthotopic liver transplantation. ${ }^{46}$ Therefore, DIC has been postulated in these patients, as elevated TAT and $\mathrm{D}$-dimer levels have been observed. It is unclear whether this fibrinolytic response is primary or secondary. Antifibrinolytic drugs, if used cautiously, result in markedly reduced bleeding, thus decreasing the need for blood and blood-product substitution. ${ }^{2}$

The LeVeen shunt is invariably related to DIC; hemorrhages seen in conjunction with peritoneovenous shunt operation are usually caused by a DIC-like presentation with a profound activation of the fibrinolytic system. This complication seems to be due to the influx of ascites fluid containing tissue factor (TF), tPA, uPA, and fibrin-related products, and so forth. $\mathrm{TF},{ }^{47}$ the activator of the extrinsic pathway in the coagulation system, is considered to be the cause of DIC.

\section{Sclerotherapy of Bleeding Esophageal Varices}

Prof. Mammen and co-workers reported hemostatic activation during sclerotherapy of bleeding esophageal varices. ${ }^{48}$ Sclerotherapy of bleeding esophageal varices has now become a common procedure in liver cirrhosis, but little had been known about the possible entry of sclerosants into the systemic circulation since 1991. A

Table 4 Laboratory Findings during Orthotopic Liver Transplantation

\begin{tabular}{lll}
\hline Test & $\begin{array}{l}\text { Anhepatic } \\
\text { Phase }\end{array}$ & $\begin{array}{l}\text { Reperfusion } \\
\text { Phase }\end{array}$ \\
\hline ELT & $\downarrow \downarrow$ & $\downarrow \downarrow$ \\
Plasminogen & $\downarrow$ & $\downarrow$ \\
PI & $\downarrow$ & $\downarrow$ \\
tPA & $\uparrow \uparrow$ & $\uparrow \uparrow$ \\
PAI-1 & $\downarrow \downarrow$ & $\downarrow \downarrow$ \\
Fibrin(ogen) split & $\uparrow$ & $\uparrow$ \\
$\quad$ products (FSPs) & & \\
FXIII & $\downarrow$ & $\downarrow$ \\
TAT & $\uparrow$ & $\uparrow$ \\
D-dimer & $\uparrow$ & \\
\hline , decreased or shortened; $\downarrow \downarrow$, markedly decreased or shortened; \\
$\uparrow$, increased; $\uparrow \uparrow$, markedly increased. \\
Source: Modified from Mammen EF. Coagulopathies of liver \\
disease. Clin Lab Med 1994;14:769-780.
\end{tabular}


mixture of thrombin, sodium tetradecyl, and cefazolin was injected to study the effect of those factors on several hemostatic parameters. In 24 patients with liver cirrhosis (Child's classification C), almost all patients had elevated D-dimer, tPA, and PAI-1 levels, but they had decreased fibrinogen, AT, PI, and PC levels, whereas TAT levels were within the normal range before the injection. Immediately after the injection, the TAT, D-dimer, and tPA levels rose significantly, whereas the PAI-1 and PC levels decreased, and the AT, PI, and fibrinogen levels remained unchanged. These data indicate that thrombin entered the systemic circulation (elevated TAT) and that the hemostasis system was briefly systemically activated (elevated D-dimer). In spite of these changes in the hemostasis system, there were no clinically detectable thrombotic or hemorrhagic complications.

\section{CONCLUSION}

Professor Eberhard F. Mammen greatly contributed to the understanding of the relationship between hemostatic abnormalities and liver diseases, that is, that the physiology of the hemostatic system is closely linked to liver function, and consequently many clinical applications have been developed partly based on his contribution.

\section{REFERENCES}

1. Mammen EF. Coagulopathies of liver disease. Clin Lab Med 1994;14:769-780

2. Mammen EF. Coagulation defects in liver disease. Med Clin North Am 1994;78:545-554

3. Mammen EF. Coagulation abnormalities in liver disease. Hematol Oncol Clin North Am 1992;6:1247-1257

4. Mammen EF. Blood coagulation disorders in liver diseases. Dtsch Med Wochenschr 1970;95:2241-2242

5. Carlier M, Van Obbergh LJ, Veyckemans F, et al. Hemostasis in children undergoing liver transplantation. Semin Thromb Hemost 1993;19:218-222

6. Matsumoto T, Wada H, Nishiyama H, et al. Hemostatic abnormalities and changes following bone marrow transplantation. Clin Appl Thromb Hemost 2004;10:341-350

7. Stafford DW. The vitamin K cycle. J Thromb Haemost 2005; 3:1873-1878

8. Aljabiri MR, Lodato F, Burroughs AK. Surveillance and diagnosis for hepatocellular carcinoma. Liver Transpl 2007; 13:S2-S12

9. Tripodi A, Chantarangkul V, Mannucci PM. The international normalized ratio to prioritize patients for liver transplantation: problems and possible solutions. J Thromb Haemost 2008;6:243-248

10. Akiyama K, Nakamura K, Makino I, et al. Antithrombin III producing hepatocellular carcinoma. Thromb Res 1993;72: 193-201

11. Koike C, Hayakawa Y, Niiya K, Sakuragawa N, Sasaki H. The production of heparin cofactor II is not regulated by inflammatory cytokines in human hepatoma cells: comparison with plasminogen activator inhibitor type-1. Thromb Haemost 1996;75:298-302

12. Mammen EF. Antithrombin: its physiological importance and role in DIC. Semin Thromb Hemost 1998;24:19-25

13. Mammen EF. Clinical relevance of antithrombin deficiencies. Semin Hematol 1995;32(Suppl 2):2-6

14. Wada H, Sakuragawa N, Shiku H. Hemostatic molecular markers before onset of disseminated intravascular coagulation in leukemic patients. Semin Thromb Hemost 1998;24: 293-297

15. Hirose K, Okajima K, Uchiba M, Nakano KY, Utoh J, Kitamura N. Antithrombin reduces the ischemia/reperfusion-induced spinal cord injury in rats by attenuating inflammatory responses. Thromb Haemost 2004;91:162-170

16. Warren BL, Eid A, Singer P, et al. KyberSept Trial Study Group: caring for the critically ill patient. High-dose antithrombin III in severe sepsis: a randomized controlled trial. JAMA 2001;286:1869-1878

17. Kienast J, Juers M, Wiedermann CJ, et al. KyberSept investigators: treatment effects of high-dose antithrombin without concomitant heparin in patients with severe sepsis with or without disseminated intravascular coagulation. J Thromb Haemost 2006;4:90-97

18. Sakuragawa N. Regulation of thrombosis and hemostasis by antithrombin. Semin Thromb Hemost 1997;23:557-562

19. Okabayashi K, Wada H, Ohta S, Shiku H, Nobori T, Maruyama K. Hemostatic markers and the sepsis-related organ failure assessment score in patients with disseminated intravascular coagulation in an intensive care unit. Am J Hematol 2004;76:225-229

20. Griffin GC, Mammen EF, Sokol RJ, Perrotta AL, Stoyanovich A, Abildgaard CF. Alpha 2-antiplasmin deficiency. An overlooked cause of hemorrhage. Am J Pediatr Hematol Oncol 1993;15:328-330

21. Koyama T, Kakishita E, Nakai Y, Okamoto E. Significance of hemostatic molecular markers during disseminated intravascular coagulation in patients with liver cirrhosis treated by endoscopic embolization for esophageal varices. Am J Hematol 1991;38:90-94

22. Kuter DJ, Begley CG. Recombinant human thrombopoietin: basic biology and evaluation of clinical studies. Blood 2002;100:3457-3469

23. Fujikawa K, Suzuki H, McMullen B, Chung D. Purification of human von Willebrand factor-cleaving protease and its identification as a new member of the metalloproteinase family. Blood 2001;98:1662-1666

24. Faeh M, Hauser SP, Nydegger UE. Transient thrombopoietin peak after liver transplantation for end-stage liver disease. Br J Haematol 2001;112:493-498

25. Fujimura Y, Matsumoto M, Yagi H, Yoshioka A, Matsui T, Titani K. Von Willebrand factor-cleaving protease and Upshaw-Schulman syndrome. Int J Hematol 2002;75:25-34

26. Ko S, Okano E, Kanehiro H, et al. Plasma ADAMTS13 activity may predict early adverse events in living donor liver transplantation: observations in 3 cases. Liver Transpl 2006; $12: 859-869$

27. Taylor FB Jr, Toh CH, Hoots K, Wada H, Levi M. Towards a definition, clinical and laboratory criteria, and a scoring system for disseminated intravascular coagulation. Thromb Haemost 2001;86:1327-1330

28. Wada H. Disseminated intravascular coagulation. Clin Chim Acta 2004;344:13-21 
29. Soria J, Soria C, Ryckewaert JJ, Samama M, Thomson JM, Poller L. Study of acquired dysfibrinogenaemia in liver disease. Thromb Res 1980;19:29-41

30. Hoak DR, Mammen EF, Banerjee SK, Kaldor G. A fully automated immuno-turbidimetric determination of fibrin (ogen) degradation products. Thromb Res 1986;44:1-10

31. Wada H, Kobayashi T, Abe Y, et al. Elevated levels of soluble fibrin or D-dimer indicate high risk of thrombosis. J Thromb Haemost 2006;4:1253-1258

32. de Moerloose P, Palareti G, Aguilar C, Legnani C, Reber G, Peetz D. A multicenter evaluation of a new quantitative highly sensitive D-dimer assay for exclusion of venous thromboembolism. Thromb Haemost 2008;100:505-512

33. Roffi L, Colloredo G, Pioltelli P, et al. Gruppo Epatologico Lombardo. Pegylated interferon-alpha2b plus ribavirin: an efficacious and well-tolerated treatment regimen for patients with hepatitis $\mathrm{C}$ virus related histologically proven cirrhosis. Antivir Ther 2008;13:663-673

34. Wilson RF, Mammen E, Walt AJ. Eight years of experience with massive blood transfusions. J Trauma 1971;11:275285

35. Jones AL, Hulett MD, Parish CR. Histidine-rich glycoprotein: a novel adaptor protein in plasma that modulates the immune, vascular and coagulation systems. Immunol Cell Biol 2005;83:106-118

36. Gando S, Iba T, Eguchi Y, et al, for the Japanese Association for Acute Medicine Disseminated Intravascular Coagulation. (JAAM DIC) Study Group: a multicenter, prospective validation of disseminated intravascular coagulation diagnostic criteria for critically ill patients: comparing current criteria. Crit Care Med 2006;34:625-631

37. Dargaud Y, Desmurs-Clavel H, Marin S, Bordet JC, Poplavsky JL, Negrier C. Comparison of the capacities of two prothrombin complex concentrates to restore thrombin generation in plasma from orally anticoagulated patients: an in vitro study. J Thromb Haemost 2008;6:962-968
38. Walenga JM, Hoppensteadt DA. Monitoring the new antithrombotic drugs. Semin Thromb Hemost 2004;30: 683-695

39. Rost S, Fregin A, Ivaskevicius V, et al. Mutations in VKORC1 cause warfarin resistance and multiple coagulation factor deficiency type 2. Nature 2004;427:537-541

40. Wadelius M, Chen LY, Lindh JD, et al. The largest prospective warfarin-treated cohort supports genetic forecasting. Blood 2008; June 23 [Epub ahead of print]

41. Wilson RF, Mammen EF, Robson MC, Heggers JP, Soullier G, DePoli PA. Antithrombin, prekallikrein, and fibronectin levels in surgical patients. Arch Surg 1986;121:635-640

42. Senzolo M, Burra P, Cholongitas E, Burroughs AK. New insights into the coagulopathy of liver disease and liver transplantation. World J Gastroenterol 2006;12:7725-7736

43. Bezeaud A, Denninger MH, Dondero F, et al. Hypercoagulability after partial liver resection. Thromb Haemost 2007;98:1252-1256

44. Porte RJ. Coagulation and fibrinolysis in orthotopic liver transplantation: current views and insights. Semin Thromb Hemost 1993;19:191-196

45. Abe Y, Wada H, Yamada E, et al. The effectiveness of measuring for fragmented red cells using an automated hematology analyzer in patients with thrombotic microangiopathy. Clin Appl Thromb Hemost 2008; July 3 [Epud ahead of print]

46. Cerutti E, Stratta C, Romagnoli R, et al. Thromboelastogram monitoring in the perioperative period of hepatectomy for adult living liver donation. Liver Transpl 2004;10:289-294

47. Wada H, Wakita Y, Shiku H. Tissue factor expression in endothelial cells in health and disease. Blood Coagul Fibrinolysis 1995;6:S26-S31

48. Fujii Y, Sugawa C, Ozawa T, Nakumura R, Brown-Castillo J, Mammen EF. Hemostasis activation during esophageal variceal sclerotherapy with thrombin in cirrhotics. Am Surg $1991 ; 57: 222-225$ 